ROCZNIKI HUMANISTYCZNE

Tom LXIX, zeszyt $12-2021$

DOI: https://doi.org/10.18290/rh216912-12

PIOTR TOWAREK

\title{
MESSE KOMPOSITIONEN ZU EHREN DER HEILIGEN ANNA. UMRISS DER PROBLEMATIK
}

Die heilige Anna, die Mutter Marias, kommt in der Heiligen Schrift nicht vor. Ihre Gestalt wird nur noch in den Apokryphen erwähnt: Protoevangelium des Jakobus, Evangelium des Pseudo-Matthäus und andere. Doch als die Wahrnehmung von Marias Heiligkeit durch die Kirche wuchs, stieg auch die Wahrnehmung der Frau, die die zukünftige Mutter Gottes zur Welt brachte. Dies zeigte sich in den Brevieroffizien und Messformularen sowie in den Kirchen, Kapellen und Altären, die der heiligen Anna geweiht waren, und somit im liturgischen Gottesdienst und der breiteren kirchlichen Kunst, die im Westen ab dem vierzehnten Jahrhundert blühte (Piechota, Rozynkowski, Welsh, D-Vasilescu).

Im Laufe der vergangenen Jahrhunderte hat sich neben der Literatur, der Malerei oder der Bildhauerei auch die Musikkultur intensiv mit der Person der heiligen Großmutter Jesu beschäftigt. Davon zeugen die musikalischen religiösen Werke zu ihren Ehren. Ein bedeutender Teil dieser Gruppe besteht aus gereimten Offizien (Analecta 106-21), ${ }^{1}$ sowie Liedern (Czapla, Górecki), darunter die ältesten Denkmäler dieser Art auf polnischem Gebiet, d.h. zwei Kompositionen von Władysław von Gielniów (Wierzbowski, Mazurkiewicz 83-142). Beliebt in der polnischen Frömmigkeit des 18. Jahrhunderts waren auch die Stundengebete, Litaneien und Rosenkranzgebete, die mit dem Namen der

Ks. dr Piotr TowareK - Höheres Theologisches Seminar in Elbląg; Postadresse: ul. Świętego Ducha 11, 82-300 Elbląg, Polen; E-mail: towarek@op.pl; ORCID: https://orcid.org/0000-00025809-5060.

Rev. Dr. PIOTR TOWAREK, Higher Theological Seminary in Elbląg; address for correspondence: ul. Świętego Ducha 11, 82-300 Elbląg, Poland; e-mail: towarek@op.pl; ORCID: https://orcid.org/ 0000-0002-5809-5060.

${ }^{1}$ G. M. Dreves weist auf die Entstehung von sechs solcher Offizien hin. 
Mutter Mariens verbunden waren (Królikowski). Darüber hinaus ihnen wurden mit der Person der Heiligen Anna auch große vokale oder vokal-instrumentale Formen, d.h. Motetten (Festa 48-50; Jeppesen 207; Burn 223-68; Meconi, Fortuna Desperata 122-25; Charpentier 4: 127-52; Charpentier 1: 154-56), ${ }^{2}$ oder bis heute erhaltene Messproprien, wie z.B. Halleluja-Akklamationen mit Strophen (Pikulik, Śpiewy Alleluja), ${ }^{3}$ oder auch Sequenzen verbunden (Pikulik, „Sekwencje polskie“ 4: 29-30 sowie 33 und 79; Pikulik, „Sekwencje polskie“ 5: 32-39; Kowalewicz 132-302). ${ }^{4}$ Diese Kompositionen, insbesondere Moutons Motette Celeste beneficium, werden in einem Artikel, den der Autor für die Veröffentlichung vorbereitet, ausführlicher behandelt (vgl. Towarek, „Wielkie formy").

In dem hier vorliegenden Artikel werden die Messen, die der heiligen Anna gewidmet sind, vorgestellt und skizziert (vgl. Towarek, „Maria Magdalena“ und „Święta Katarzyna Aleksandryjska“; Parzych-Blakiewicz). Eine unschätzbare Hilfe bei der unternommenen Arbeit war die ausgezeichnete Monographie von Michael Alan Anderson mit dem Titel St. Anne in Renaissance Music: Devotion and Politics, in der wir nicht nur die Beschreibung der musikalischen Werke zu Ehren der heiligen Großmutter Jesu finden, sondern auch die ungewöhnlichen Umstände ihrer Entstehung entdecken, d.h. den Alltag der königlichen Höfe Europas, die Politik und die Bemühungen um die Zeugung des Thronfolgers, die mit dem Bittgebet zu St. Anne verbunden waren. Auch die für diese Studie wertvolle Monographie von Honey Meconi Pierre de la Rue and Musical Life at the Habsburg-Burgundian Court vervollständigt dieses Bild. Die Skizzen von Anderson und Meconi beschränken sich jedoch nur auf die Zeit der Renaissance. Daher die vom Autor unternommene Suche, die dazu führte, dass er weitere der heiligen Anna gewidmete Messkompositionen fand und vorstellte, die in den folgenden Jahrhunderten bis in die Gegenwart geschaffen wurden.

${ }^{2}$ Z.B. Motetten: Felix Anna (C. Festa, L. Senfl); Ave mater matris Dei (N. Gombert, J. de Mantua, J. Lhéritrier, O. di Lasso); Celeste beneficium (J. Mouton); Gaude felix Anna oraz Canticum Annae: Exultavit cor meum (M.-A. Charpentier).

${ }^{3}$ Pikulik fand 10 Akklamationen zu Ehren der heiligen Anna (Nr. 3, 4, 5, 31, 41, 66, 67, 97, 121, 122).

${ }^{4}$ Z.B. 5 Sequenzen, die im Krakauer Kreis im 15. Jahrhundert und in der ersten Hälfte des 16. Jahrhunderts geschrieben wurden, darunter 4 mit Melodien, die Pater Pikulik veröffentlichte. 
Die erste bekannte Messe, die mit dem Namen der Mutter Marias verbunden ist, ist die Missa de Sancta Anna von Pierre de La Rue (Poźniak). ${ }^{5}$ Nach Meconi könnte dieses Werk zwischen 1506 und 1516 für einen 4-stimmigen Chor komponiert worden sein (Meconi 98, 185 sowie 270). Die Messe ist in ihrer vollständigen Form (Kyrie, Gloria, Credo, Sanctus, Agnus Dei) nur in einem Wiener Manuskript VienNB Mus. 15496, mit einer Widmung an Erzherzog Karl (den späteren Kaiser Karl V.), überliefert. Die Sammlung enthielt Messen von la Rue als Hofkomponist für Karl von Habsburg, die möglicherweise für seine neue Hofkapelle in Brüssel bestimmt waren. Die Messe an St. Anna ist auch in zwei anderen Sammlungen (MontsM 773 und JenaU 7) teilweise erhalten, die für den Hof Philipps des Schönen in Burgund bestimmt waren (Meconi 19-40).

Die Missa de Sancta Anna wird sehr oft als ein Beispiel für de la Rues zugänglichsten musikalischen Stil angeführt. Viele Forscher weisen darauf hin, dass der charakteristische Modus F und die ausdrucksstarke Bewahrung der Kadenzen des Werkes nicht nur den Menschen der Renaissance, sondern auch dem Ohr des modernen Musikhörers gefällig sind. Die allgegenwärtige Verwendung von Duett-Texturen (ein starkes Merkmal von J. des Prés) und zahlreiche Fragmente, die um parallele Akkorde herum aufgebaut sind (ebenfalls charakteristisch für J. Obrecht), verleihen de la Rues Kontrapunkt eine sehr leichte Textur. Andererseits behält die Melodik des Werkes die für den Komponisten charakteristische rhythmische Lebendigkeit bei, besonders in den langen Teilen von Kyrie und Agnus Dei. Eine Besonderheit der besprochenen Messe ist die Platzierung der Motette $O$ salutaris hostia an der Stelle des Hosanna in der Sanctus-Akklamation (Anderson 67). Diese Substitution, die z.B. in einigen Mailänder Messen üblich war, sollte im Höhepunkt der Messe, d.h. im Moment der Erhebung und Überreichung der Hostie an die Gläubigen, zu noch tieferer Besinnung und Anbetung führen (la Rue, Opera omnia 2 lxvii-lxxii sowie 9 lvi-lx; Meconi, Pierre de la Rue 124). ${ }^{6}$

${ }^{5}$ P. de la Rue (1460-1518), fränkisch-flämischer Komponist und Sänger; in den Jahren von 1481 bis 1485 Tenor im Chor des Doms von Siena und von 1489 bis 1492 in 's-Hertogenbosch; 1492 bis 1506 Mitglied der burgundischen Grand Chapelle. Nach dem Tod des Prinzen Philipps IV. des Schönen (1506) blieb er einige Zeit in den Diensten seiner Frau, der Prinzessin Juana; 1508 kehrte er in das burgundische Ensemble am Hof von Margarete von Österreich in Mechelen zurück und war in den Jahren 1514-1516 im privaten Ensemble des Erzherzogs Karl von Habsburg tätig.

${ }^{6}$ Es gibt eine anhaltende ungelöste Debatte unter den Gelehrten über Pierre de la Rues Werk. Einige glauben, dass $O$ salutaris Hostia Teil der Messe an St. Anna ist, während andere der Meinung sind, dass es eine unabhängige Komposition ist. Siehe die kritische Ausgabe der Werke des Komponisten mit Kommentar. 
Es ist erwähnenswert, dass das Wiener Manuskript der Messe als einziges in der Tenorstimme das lateinische Incipit Felix Anna enthält, was auf eine melodische Quelle hinweisen würde, nämlich die Antiphon aus dem Brevier-Offizium der Heiligen Anna (Anderson 80). Es sind jedoch keine weiteren Belege für diesen Tenor-Cantus firmus erhalten geblieben. Es ist kein Musikwerk gefunden worden, das der Messe zugrunde gelegen haben könnte (Anderson 283). ${ }^{7}$ Es kehren jedoch mindestens drei verschiedene melodische Phrasen im gesamten Werk wieder, die in allen Stimmen vorkommen. Der erste von ihnen enthält sogar das Hauptmotiv, das jeden Satz eröffnet (gewöhnlich in Tenor und Cantus). Wie einige Autoren bemerken, leisten diese melodischen Fragmente einen Beitrag zur Einheit der Klangwelt von Pierre de la Rues Messe zu Ehren von St. Anne (Dickey).

\section{„MISSA SANCTA ANNA“-MARCIN MIELCZEWSKI}

Ein Beispiel für eine Messe, die der heiligen Anna gewidmet ist, findet sich auch in der Barockzeit der polnischen Musikkultur. Es handelt sich um die Missa Sancta Anna von Marcin Mielczewski, ${ }^{8}$ die im Jahre 2003 ihre erste kritische Ausgabe erhielt (Mielczewski 137-202). ${ }^{9}$ Sie gehört zu der Sammlung konzertanter Messen des Komponisten, von denen außer des der heiligen Anna gewidmeten Werkes, nur die Missa O Gloriosa Domina (Feicht) in ihrer Gesamtheit (d.h. als kompletter Zyklus) bis in unsere Zeit erhalten geblieben ist. Dagegen zwei weitere, die Missa Triumphalis und die Missa Cerviensiana, sind nur fragmentarisch und als unvollständiger zweiteiliger Zyklus erhalten geblieben (Hinz 118).

${ }^{7}$ Wie Anderson anmerkt, hat keine der drei zeitgenössischen Ausgaben von le Rues Messe über die heilige Anna überhaupt eine Untersuchung des Ursprungs der Messe vorgenommen.

${ }^{8}$ M. Mielczewski (gest. 1651), polnischer Komponist und Kapellmeister; Geburtsdatum und -ort unbekannt; einer der bekanntesten Vertreter der polnischen Musikkultur des 17. Jahrhunderts in Europa, tätig in den Kapellen der polnischen Vasadynastie; er war Musiker von König Ladislaus IV. (möglicherweise auch von Sigismund III. Vasa), und später Kapellmeister in der Kapelle des Fürsten Karl Ferdinand Vasa - Bischof von Plock und Breslau.

${ }^{9}$ Bis zum letzten Krieg wurde das Manuskript der Missa Sancta Anna in der Sammlung der Stadtbibliothek in Gdańsk aufbewahrt (Ref. Cath. Q. 10). Sie ging jedoch während des Krieges verloren. Unter der gleichen Signatur wurde auch die Partitur der Messe aufbewahrt (die Herausgabe von Crato Bünter). Bis heute sind nur Fotokopien von Manuskripten, die vor dem Krieg für Z. Jachimecki angefertigt wurden (nicht von allen Seiten), sowie Fotokopien der ersten drei Seiten der Partitur erhalten geblieben. Die Zusammenstellung und Rekonstruktion der fehlenden Teile wurde von Z. M. Szweykowski vorgenommen. 
Die reiche Besetzung der Missa Sancta Anna besteht aus einem sechsstimmigen Chor (2CA2TB), einem Coro cappella in gleicher Besetzung und einem sechsstimmigen Instrumentenensemble (zwei Violinen, drei Posaunen und Fagott). In einigen Manuskripten Danziger Provenienz wurde zu dieser Zeit ein Instrumentalensemble hinzugefügt, um einige der vorhandenen Stimmen, sowohl vokal als auch instrumental, zu verdoppeln. Sie stellen ein Zeugnis der Aufführungspraxis in Gdańsk dar (Szweykowski 7).

Die Missa Sancta Anna repräsentiert einen Typ polychöriger Technik, der bereits zu Beginn des 17. Jahrhunderts entwickelt wurde und tatsächlich weit von der klassischen Form entfernt ist. Es geht weniger um den Wandel von Klanggruppen als um die Räumlichkeit der Performance. Der zweite Chor, in diesem Fall coro cappella genannt, spielt keine eigenständige Rolle, sondern schließt sich von Zeit zu Zeit dem Haupt-, d.h. ersten Chor (besetzt mit einer Gruppe von Solisten) an und verdoppelt seine Stimmen.

In seiner Messe zu Ehren der heiligen Anna verwendete Mielczewski auf seiner charakteristischen Art und Weise das damals bekannte polnische religiöse Lied O Maryia, cna dziewica (Szweykowski 17). Seine Melodie findet sich im Manuskript Nr. 10002 der Jagiellonen-Bibliothek, wo es zweimal geschrieben ist: auf Seite 1r unisono ohne signierten Text (als zweites Lied) und auf Seite 21 r mit Bassbegleitung, mit signiertem Text (als zweiundneunzigstes Lied). Wie Szweykowski anmerkt, wurde im Jagiellonen-Manuskript die Melodie O Maryia, cna dziewica im geraden Metrum notiert. Mielczewski hingegen hat es in seinem Werk um eine Sekunde nach oben transponiert, einige leichte melodische Veränderungen vorgenommen und es im Verlauf der Messe sowohl im geraden als auch im ungeraden Metrum notiert (Szweykowski 17). Leider kennen wir die Umstände nicht, unter denen die Missa Sancta Anna komponiert wurde.

\section{MESSEN DER ROMANTIK}

Die Person der heiligen Anna wurde auch von Komponisten der Romantik beachtet. Ein Beispiel ist die Erste Sonntagsmesse in honorem St. Annae (Op. 15) von Josef Gruber. ${ }^{10}$ Die Messe besteht aus sechs Sätzen: Kyrie, Gloria, Credo, Sanctus, Benedictus, Agnus Dei (Gruber). Es ist für einen gemischten vierstim-

${ }^{10}$ J. Grubner (1855-1933), österreichischer Komponist, Organist, Pädagoge, Schüler A. Bruckners, verbunden mit der cäcilianischen Bewegung; Organist am Kloster St. Florian, Professor für Musik am Institut für Lehrerbildung und Kantor an der Kapuzinerkirche in Linz; Autor von über 300 religiösen Kompositionen, darunter 58 Messen und 17 Requiems. 
migen Chor (SATB) besetzt, der von einem Instrumentenensemble (Violine I, Violine II, Cello und Bass, Horn I, Horn II) und Orgel begleitet wird, die den Chor auch selbständig begleiten kann. Als Cäcilianer verwendete der Komponist in der Messe die Melodik des Gregorianischen Chorals. Solche Choral-Zitate sind deutlich in vier Teilen Credo zu hören: Patrem omnipotentem, Et resurrexit, Et incarnatus est, Et unam sanctam Catholicam (Helfgott 467-68). Ein ähnliches Beispiel ist die Missa in honorem Sanctae Annae aus dem Jahr 1933 von Johan (Winnubst). ${ }^{11}$ Das Werk wurde ein Jahr vor dem Tod des Komponisten geschrieben und war für drei gleichberechtigte Stimmen mit Orgelbegleitung (ad tres voces aequales organo comitante) bestimmt.

Eine Widmung zu Ehren der heiligen Anna findet sich auch in den Werken von Kazimierz Garbusiński (Perkowska 231) ${ }^{12}$ aus dieser Zeit. Sie wurden im Jahr 1913 komponiert und für zwei dreistimmige Männerchöre a'capella Missa in honorem S. Annae (Op. 17) besetzt. Seit 1909 war der Komponist mit der St. Anna-Kirche in Krakau verbunden, wo er als Organist tätig war. Die Messe über die der Heiligen Anna muss also etwas mit dem Titel der Krakauer Kirche zu tun gehabt haben. An diesem Ort wurde später die Oratoriengesellschaft gegründet, die der Komponist leitete (Garbusiński).

Spuren spätromantischer musikalischer Bezüge zur Heiligen Anna finden sich auch in der Schweiz. Eine davon ist eine 1909 komponierte Missa in honorem Sanctae Annae von Charles Haenni. ${ }^{13}$ Das Stück ist für gemischten Chor (SATB) konzipiert und besteht aus vier Teilen: Kyrie, Gloria, Sanctus, Agnus Dei (Allet-Zwissig 243-51). Das zweite schweizer Beispiel ist die Missa in honorem Sanctae Annae von Richard Flury. ${ }^{14}$ Der Komponist hat es für einen dreistimmigen gemischten Chor und Violinsolo (ad libitum) unter Beteiligung der Orgel und des Streicherensembles (Violinen I-II, Viola) vorgesehen. Die

${ }^{11}$ J. Winnubst (1885-1934), niederländischer Organist und Chorleiter; studierte Orgel, Kirchenmusik und Komposition in Amsterdam; ab 1910 Organist und Chorleiter an der Kathedrale von Utrecht, ab 1925 Organist, Chorleiter und Lehrer für Orgel und Komposition an der St. Cecilia Kerkmuziek School in Utrecht; Kapellmeister des Utrechter Erzbischofs Henricus van de Wetering.

${ }^{12}$ K. Garbusiński (1883-1945), polnischer Komponist, Organist, Dirigent; Schüler von Z. Noskowski, W. Żeleński, F. Nowowiejski; Gründer der Oratoriengesellschaft (1919-1924), ab 1918 Professor des Konservatoriums in Krakau; Kompositionen: Oratorien (Siedem stów Chrystusa na Krzyżu, Św. Franciszek z Asyżu), 16 Messen, Requiem, Hymnen, Kantaten, Lieder, Bearbeitungen von Weihnachtsliedern, Orgelvorspiele.

${ }^{13}$ Ch. Haenni (1867-1953), Schweizer Musiker und Komponist aus dem Wallis; Studium in Genf und Straßburg; ab 1906 Organist an der Kathedrale in Sitten; Kompositionen: Messen, geistliche und weltliche Lieder, Orgelwerke.

${ }^{14}$ R. Flury (1896-1967), Schweizer Komponist und Dirigent; studierte in Genf und Bern, kehrte 1919 in seine Heimatstadt Biberist zurück und unterrichtete an der Städtischen Musikschule Solothurn, ab 1931 an der Solothurn-Kanton-Schule; Kompositionen: 3 Opern, 7 Sinfonien, Solokonzerte, Quartette, Sonaten, Messen. 
bescheidene Besetzung der Messe wurde vermutlich von den Aufführungsmöglichkeiten diktiert, da das Werk für einen kleinen Gemeindechor in Gänsbrunnen (Kanton Solothurn) komponiert wurde. Bei der Uraufführung des Werkes (am 26. Juli 1964) spielte der Sohn des Komponisten, Urs Joseph Flury, die solistische Violine (Schläpfer 22).

\section{BRETONISCHE MESSEN}

Unter den musikalischen Widmungen, die mit der Heiligen Anna verbunden sind, nimmt eine Gruppe zeitgenössischer bretonischer Kulturkreis-Messen des 20. Jahrhunderts einen wichtigen Stellenwert ein. Die Geschichte ihrer Entstehung ist mit dem Heiligtum der Offenbarungen der Heiligen Anna in Sainte-Anne-d'Auray ${ }^{15}$ verbunden.

Das erste Beispiel ist die Messe breve en l'honneur de Sainte Anne, deren Autor Joseph-Guy Ropartz ist (Bodlore-Penlaez 74-81). ${ }^{16}$ Das im Jahre 1921 komponierte Werk ist für drei gleiche Stimmen (Sopran, Mezzosopran, Alt) und Orgel (3 Voix Egales et Orgue) besetzt. Die Messe besteht aus fünf Teilen: Kyrie, Gloria, Sanctus, Benedictus, Agnus Dei und ist eine Würdigung des Komponisten an die in der Bretagne so verehrte Heilige Anna. Es verweist auch auf die lokale Musikkultur, weil wir in seinen Teilen die Melodien bretonischer Lieder finden (Bempéchat 20).

Ein weiteres Beispiel für eine bretonische Messe, die der heiligen Anna gewidmet ist, ist die Messe en l'honneur de Sainte Anne von Jef Le Penven (Bodlore-Penlaez 66-69; Dumerchat-Schouten 34-40). ${ }^{17}$ Die Messe ist für einen gemischten Chor, ein Ensemble und zwei Orgeln besetzt (pour choeurs mixtes, Assemblée et deux Orgues). Die Komposition enthält nicht nur feste Messens-

${ }^{15}$ Sainte-Anne-d'Auray (bret. Santez-Anna-Wened) - ein bretonisches Sankt-Annen-Heiligtum im Nordwesten Frankreichs, erbaut im 17. Jahrhundert. Es wurde im 17. Jahrhundert als Ergebnis von Offenbarungen, die I. Nicolazic erlebte, gebaut. Der Ort wurde 1996 von Papst Johannes Paul II. besucht, der an die Botschaft der heiligen Anna erinnerte.

${ }^{16}$ J. G. Ropartz (1864-1955), französischer Komponist und Dirigent bretonischer Herkunft; Ausbildung am Jesuitenkolleg seiner Heimatstadt, Studium der Rechtswissenschaften in Rennes und ab 1885 am Pariser Konservatorium (Komposition, Orgel); von 1894 bis 1919 Direktor des Konservatoriums in Nancy; von 1919 bis 1929 Direktor des Straßburger Konservatoriums. Kompositionen: 5 Sinfonien, Orchesterwerke, Kammermusikwerke, Klavierwerke; Oper La pays, Ballett, Theatermusik, religiöse Werke: Messen, Requiem, Chorwerke (Kantaten, Lieder), Psalmen für Chor und Orchester.

${ }^{17}$ J. L. Penven (1919-1967), französischer Komponist und Dirigent bretonischer Herkunft; studierte am Pariser Konservatorium; leitete ab 1940 das Orchestre de Bretagne in Rennes; künstlerisch mit der Bretagne verbunden. Neben der Messe zu Ehren der heiligen Anna, komponierte er: Messe en l'honneur de St Ivy oraz Messe de Noël. 
ätze (Kyrie, Gloria, Sanctus, Benedictus, Agnus Dei), sondern auch variable Teile (Messeproprien). Als Introitus wird das beliebte bretonische Lied zu Ehren der heiligen Anna Santéz Anna, gouarnet ho Pretoned verwendet, das von Le Penven (Aveidom peh ur gloér) harmonisiert wurde. Als Offertorium der Messe verwendete der Komponist ein Orgelstück, als Communio den bretonischen Hymnus $O$ Anna mam 'Marie. Die Messe beinhaltet auch ein Lied, das ein Gebet für die während der Kriege gefallenen Bretonen ist: Peden aveit en du maru ér brezél (Prière pour les Morts à la guerre). Es handelt sich um eine Komposition von Jean Jouan, die in der Regel in ihrer französischen Fassung (Tes fils bretons morte pour la France) im Wallfahrtsort Sanite-Anne-d'Auray am Fuße der Statue der Heiligen Anna anlässlich verschiedener Gedenkfeiern gesungen wird. Die ursprüngliche Version des Gesangs funktioniert im so genannten gwenedeg, also Vanethean-Dialekt, einer der Abarten der bretonischen Sprache (siehe Santez Anna, o Mamm garet). Die Messe wurde mit zwei instrumentalen Klammern belegt: einem Orgelpräludium zu Beginn und einem Orgelpostludium, das das Ganze abschließt. Bei der Aufnahme des Werkes, die im Jahre 1960 in der Wallfahrtskirche und im Basilikum von Sainte-Anned'Auray stattfand, trat der Chor des örtlichen Priesterunterseminars (la Chorale du Petit Séminaire de Sainte-Anne-d'Auray) auf, wobei Le Penven und Pater Lefrère die beiden Orgeln spielten (siehe Messe en 1'honneur de Sainte Anne).

Der Kult der Heiligen Anna bleibt in der französischen Bretagne auch im laufenden Jahrhundert lebendig. Das wird durch eine im Jahr 2019 komponierte Messe bretonne en l'honneur de Sainte Anne et Saint Yves bewiesen. Der Name des heiligen Yves Hélory $(+1303)$, der neben der heiligen Anna auch der Schutzpatron der Bretagne ist, wurde mit diesem Werk in Einklang gebracht. Der Autor der Messe ist Louis-Marie Salaün (Composition d'une Messe bretonne) ${ }^{18}$ der sie zwei berühmten Bretonen widmete: dem Dichter Yann-Ber Kalloc'h $(+1917)$ und dem Priester Yann-Vari Perrot $(+1943)$. Der erste war mit dem Wallfahrtsort Sainte-Anne-d'Auray und der zweite mit der bretonischen Nationalbewegung verbunden. Die vierteilige Messe (Kyrie, Alleluja, Sanctus, Agnus Dei) wurde im Hinblick auf ihre praktische Verwendung in der Pfarrliturgie der bretonischen Diözesen komponiert. In der Melodik des Werkes finden sich direkte Bezüge zum gregorianischen Choral, aber auch

${ }^{18}$ L.-M. Salaün (geb. 1982), bretonischer Musiker; von 2005 bis 2010 Kantor in der Diözese Puyen-Velay; 2012-2013 Leiter des Chors von Notre-Dame de la Trinité in Blois; 2015-2018 Dirigent des Chors von Saint Nicolas in Troyes; ab 2019 Mitglied des gregorianischen Ensembles der Kathedrale von Troyes; seit 2017 Mitarbeiter des bretonischen Blogs Ar Gedour: Actualite culturelle et spirituelle en Bretagne. 
zu bretonischen Hymnen und Liedern zu Ehren der Heiligen Anna und des Heiligen Yves. Die Absicht des Komponisten war, dass die einstimmige Messe jenen Gemeinden dienen konnte, die keinen Chor oder keine ausreichende Anzahl von Sängern hatten, um die mehrstimmigen Kompositionen zu singen. Dennoch wurde empfohlen, dass die gesungenen Teile der Messe während der Liturgie von der Orgel begleitet werden, mit entsprechender Harmonisierung. Darüber hinaus haben die Verantwortlichen für das liturgische Gesangsrepertoire in den bretonischen Diözesen vorgeschlagen, dass für Sonntage oder Feierlichkeiten, die die Darbietung von Gloria in der Höhe und Ich glaube an einen Gott erfordern, gregorianische Gesänge, die den Gläubigen recht vertraut sind, zu den Teilen der Messe hinzugefügt werden sollten.

In der Gruppe der zeitgenössischen bretonischen Messen, die der heiligen Anna dargebracht werden, befindet sich noch die Messe de Sainte-Anne, deren Autor Christophe Le Marec ist (L'Académie de Musique). ${ }^{19}$ Die Messe besteht aus vier ständigen Teilen (Kyrie, Gloria, Sanctus, Agnus Dei) und ist für einen vierstimmigen gemischten Chor (SATB) konzipiert.

\section{ST. ANNA-MESSE - PETER F. SCHNEIDER}

Ein Beispiel für einen zeitgenössischen musikalischen Bezug auf die Person der Heiligen Anna findet sich auch in Deutschland, nämlich die St. AnnaMesse, komponiert von Peter F. Schneider (siehe Über mich). ${ }^{20}$ Der eigenen Aussage des Komponisten nach soll die Entstehung des Werkes mit einem Treffen von Jugendlichen im Kloster der Schwestern der Kongregation der heiligen Anna in Kochel am See in Bayern ${ }^{21}$ zusammenhängen. Daher auch der Name: St. Anna-Messe. ${ }^{22}$ Schneiders Messe besteht aus sechs deutschsprachigen festen Teilen: Zum Kyrie: Jesus Christus Davids Sohn, Zum Gloria:

${ }^{19}$ Ch. L. Marec - französischer Komponist, Dirigent, Professor für Musikpädagogik am Collège Jules Simon in Vannes (Morbihan), Direktor der Académie de musique et d'arts sacrés, die seit 1999 in der Wallfahrtskirche von Sainte-Anne-d'Auray tätig ist.

${ }^{20}$ P. F. Schneider (geb. 1959) in Sasbach bei Freiburg, deutscher Pädagoge und Komponist, Absolvent des Musikstudiums in München, studierte Komposition bei B. Rövenstrunck; leitet Seminare und Fortbildungen für Kirchenmusiker, Religions- und Musiklehrer.

${ }^{21}$ Die Ordensgemeinschaft der Schwestern von der heiligen Anna wurde 1922 von Anna Adelmann in München gegründet. Das Mutterkloster in Kochel am See ist seit 1926 in Betrieb.

22 „Die St. Anna-Messe wurde von mir, Peter F. Schneider, komponiert. Der Beginn der Komposition war bei einem Jugendtreffen, das in Kochel am See in Bayern im Haus der St. Anna-Schwestern stattfand. So bekam die Messe den Namen St. Anna-Messe“ (E-Mail-Korrespondenz mit dem Komponisten vom 26. Februar 2021). 
Ehre sei Gott in der Höhe, Zum Credo: Ich glaube an Gott, Zum Sanctus: Heilig, Heilig, Heilig, Zum Agnus Dei: Oh du Lamm Gottes. Der Komponist plante es als ein Gebrauchsstück, das als Teil der Liturgie abwechselnd von einem vierstimmigen Chor (SATB) oder Kantor und der Gemeinde aufgeführt wird, begleitet von Orgel oder Klavier oder Gitarre (nach eigener Wahl). Die St. Anna-Messe ist dank ihrer Klarheit, Frische und universellen Ausstrahlung sehr beliebt geworden. Ihre Form ermöglicht es den Teilnehmern der Liturgie, mitzusingen, und schafft gleichzeitig eine festlichere Atmosphäre (Schneider).

\section{AUFSUMMIERUNG}

Der sich in der Kirche entfaltende Kult der Heiligen Anna beeinflusste die Wiederbelebung der mit ihrem Namen verbundenen liturgischen Musik, vor allem im 15. und 16. Jahrhundert. Dies ist die Entstehungszeit der zu Beginn dieser Studie angedeuteten Messeproprien: Alleluja-Akklamationen mit Strophen und Sequenzen und vor allem zahlreiche Motetten. (Festa, Senfl, Mouton, de Mantua, Lhéritrier, di Lasso). Der Name der Heiligen Anna wurde auch mit der Form der Messe verbunden. Diese reichen von der Renaissance Missa de Sancta Anna von Pierre de la Rue, über die Barockmesse von Marcin Mielczewski bis hin zu den romantischen Werken von Josef Gruber, Kazimierz Garbusiński, Charles Haenni oder Richard Flury. Als interessante Entdeckung erwiesen sich Angaben zur Musikkultur der französischen Bretagne, darunter Messen des 20. Jahrhunderts und zeitgenössische Messen, die der heiligen Anna gewidmet sind und mit dem bretonischen Heiligtum in SainteAnne-d'Auray in Verbindung gebracht werden. Sie sind durchdrungen vom gregorianischen Gesang und der bretonischen Musiktradition, insbesondere vom religiösen Gesang (Ropartz, Le Penven, Salaün, Le Marec). Diese Entdeckung ist erfreulich, ebenso wie das zeitgenössische deutschsprachige Werk von Peter F. Schneider, St. Anna-Messe. Ebenso erfreuen wir uns an wenig bekannten Kompositionen polnischer Provenienz. Die im Jahr 2000 von Józef Świder komponierte Hymne an die Heilige Anna (Hymn do św. Anny), die auf dem Text des altpolnischen anonymen Liedes Ja sobie wybratem na obrone, Babkę Chrysta Pana, święta Annę (Kochańska 185, Darłak 92) basiert, oder das Stück Felix Anna von Anna M. Huszcza aus dem Jahr 2015 (Felix Anna). Dies sind nicht nur kulturelle Bezüge zur Person der heiligen Anna, sondern vor allem wichtige Zeugnisse für die Vitalität des Christentums und des Heiligenkultes im 21. Jahrhundert. 


\section{BIBLIOGRAFIE}

Allet-Zwissig, Danielle, u. Katia Chevrier. Charles Haenni, musicien et compositeur valaisan (1867-1953). Archives de 1'Etat du Valais, 2005.

Analecta hymnica medii aevi, Bd. 5: Historiae rhythmicae, hrsg. von Guido M. Dreves, Fuess Verlag (R. Reisland), 1886.

Anderson, Michael Alan. St. Anne in Renaissance Music: Devotion and Politics. Cambridge UP, 2014.

„Aveidom peh ur gloér.“ Canitiques et musique de sacrée de Bretagne, www.kan-iliz.com/sainteanne-aveidom-peh-ur-gloer. Abruf am 12.02.2021.

Bempéchat, Paul-André. „Toward a Breton Musical Patrimony: Symbiosis and Synthesis of the Folkloric, the Classical and the Impressionistic." Proceedings of the Harvard Celtic Colloquium, Bd. 22, 2002, S. 1-38.

Bodlore-Penlaez, Mikael, u. Aldo Ripoche. Musique classique bretonne - Sonerezh klasel breizh. Coop Breizh, 2013.

Burn, David J. „Ludwig Senfl and the Mass Proper: Aspects of Chronology.“ Senfl-Studien 1, hrsg. von Stefan Gasch et al., Verlag Hans Schneider, 2012, S. 223-268.

Charpentier, Marc-Antoine. Petits Motets, Bd. 1: Motets à une et deux voix, hrsg. Von Catherine Cessac, Éditions du Centre de Musique Baroque de Versailles, 2009.

Charpentier, Marc-Antoine. Petits Motets, Bd. 4: Motets à 3 voix et instruments II - a. motets avec voix de dessus, hrsg. von Shirley Thomson, Éditions du Centre de Musique Baroque de Versailles, 2018.

„Composition d'une Messe bretonne en l'honneur de Sainte Anne et Saint Yves.“ Ar Gedour: Actualite culturelle et spirituelle en Bretgne, www.argedour.bzh/composition-dune-messe-bretonne-enlhonneur-de-sainte-anne-et-saint-yves. Abruf am 16.02.2021.

Czapla, Antoni. Polskie pieśni ku czci św. Anny w modlitewnikach górnoślaskich od początku XIX w. do 1914 r. Biblioteka WTL, Uniwersytet Śląski, 1976. Unveröffentlichte MA-Arbeit.

Darłak, Grażyna. „Inspiracje religijne w twórczości Józefa Świdra.“ Wartości w muzyce, Bd. 5, 2013, S. 85-89.

Dickey, Timothy. „Pierre de la Rue: Missa de Sancta Anna.“ Allmusic, www.allmusic.com/composition/ missa-de-sancta-anna-mc0002438284. Abruf am 20.11.2020.

Dumerchat-Schouten, Anne-Marie. „Jef Le Penven et le chant populaire breton: Des sources à la composition." Musique bretonne, Nr. 232, 2012, S. 34-40.

Ene D-Vasilescu, Elene, Hrsg. Devotion to St. Anne in Texts and Images. From Byzantium to the Late European Middle Ages. Palgrave Pivot, 2018.

Feicht, Hieronim. „Marcin Mielczewski: Missa super ,O gloriosa Domina“،“ Opera Musicologica Hieronymi Feicht, Bd. 3: Studia nad muzyka polskiego renesansu i baroku, hrsg. von Z. Lissa et al., Polskie Wydawnictwo Muzyczne, 1980, S. 455-471.

„Felix Anna. Koncert Galowy Jubileuszu 50-lecia Warszawskiego Chóru Międzyuczelnianego.“ Warszawski Chór Międzyuczelniany, www.wchm.pl/aktualnosci/17-wydarzenia/11-felix-anna. Abruf am 20.02.2021.

Festa, Costanzo. Opera Omnia, hrsg. von Albert Seay, Bd. 5: Motetti III, American Institute of Musicology, 1979. Corpus Mensurabilis Musicae 25.

Garbusiński, Casimiro. Missa in honorem S. Annae: Pro duobus trivocalis choris virilibus a capella. Wydawnictwo Muzyczne i Księgarnia B. J. Zalewski, 1913. 
Helfgott, Maria. Die Orgelmesse. Eine Untersuchung der orgelbegleiteten Messen vom ausgehenden 18. bis zum beginnenden 20. Jahrhundert. Universität Wien, 2009. Dissertation.

Hinz, Edward. Zarys historii muzyki kościelnej. Wydawnictwo Bernardinum, 2000.

Gruber, Josef. Erste Sonntagsmesse. St. Anna-Messe. Op. 15 fur Soli ad lib., vierstimmig gemischten Chor und Orgel. Dr. J. Butz - Musikverlag St. Augustin 1990.

Jeppesen, Knud. The Style of Palestrina and the Dissonance. Dover Publications, 1970. Reprint 2005.

„Winnubst Johan.“ Composers - Classical - Music, www.composers-classical-music.com/w/ WinnubstJohan.htm. Abruf am 4.12.2020.

Kochańska, Anna. „Józefa Świdra twórczość o tematyce religijnej w kontekście twórczości kompozytorów śląskich.“ Muzyka Religijna - Między Epokami i Kulturami, Bd. 1, 2008, S. 184-189.

Kowalewicz, Henryk. „Polska twórczość sekwencyjna wieków średnich.“ Średniowiecze. Studia o kulturze, Bd. 2, hrsg. von Julian Lewański, Ossolineum, 1965, S. 132-302.

Królikowski, Janusz, Hrsg. Ku ratunkowi memu pokwap się. Godzinki i inne modlitwy do świętych w polskiej pobożności XVIII wieku. Biblos, 2009.

„L'Académie de Musique et d'Arts Sacrés.“ Diocèse de Vannes, www.vannes.catholique.fr/ lacademie-de-musique-et-darts-sacres. Abruf am 12.02.2021.

Mazurkiewicz, Roman. Polskie średniowieczne pieśni maryjne. Studia filologiczne. Wydawnictwo Naukowe Akademii Pedagogicznej, 2002.

Meconi, Honey. Pierre de la Rue and Musical Life at the Habsburg-Burgundian Court. Oxford UP, 2003.

Meconi, Honey, Hrsg. Fortuna Desperata: Thirty-six Settings of an Italian Song. A-R editions, 2001.

„Messe en l'honneur de Sainte Anne.“ BnF.Gallica. Bibliothèque Nationale de France, www.gallica.bnf.fr/ ark:/12148/bpt6k88032821/f2.media. Abruf am 12.02.2021.

Mielczewski, Marcin. „Missa Sancta Anna.“ Opera Omnia, Bd. 3: Msze koncertujace, hrsg. von Z. M. Szweykowski, Instytut Sztuki Polskiej Akademii Nauk, Musica Iagellonica, 2003, S. 137-202.

Parzych-Blakiewicz, Katarzyna. „Warmińska hagiologia (2010-2018).“ Studia Elbląskie, Nr. 20, 2019, S. 285-300.

Perkowska, Małgorzata. „Garbusiński Kazimierz.“ Encyklopedia muzyczna PWM. Część biograficzna, Bd. 3: E-F-G, hrsg. von E. Dziębowska, Polskie Wydawnictwo Muzyczne, 1987, S. 231.

Piechota, Zbigniew. „Średniowieczne śląskie teksty liturgiczne o św. Annie.“ Studia z dziejów liturgii w Polsce, hrsg. von Marian Rechowicz, Wacław Schenk, Towarzystwo Naukowe KUL, 1976, S. $127-165$.

Pierre de la Rue. Opera omnia, hrsg. von Nigel St. John Davison, et al., Bd. 2: Five Masses, American Institute of Musicology, 1992. Corpus Mensurabilis Musicae 97.

Pierre de la Rue. Opera omnia, hrsg. von Nigel St. John Davison et al., Bd. 9: The Motets, American Institute of Musicology, 1996. Corpus Mensurabilis Musicae 97.

Pikulik, Jerzy. „Sekwencje polskie.“ Musica Medii Aevi, hrsg. von Jerzy Morawski, Bd. 4, Polskie Wydawnictwo Muzyczne, 1973, S. 7-128.

Pikulik, Jerzy. „Sekwencje polskie.“ Musica Medii Aevi, hrsg. von Jerzy Morawski, Bd. 5, Polskie Wydawnictwo Muzyczne, 1976.

Pikulik, Jerzy. Śpiewy Alleluja o świętych w polskich rękopisach przedtrydenckich. Wydawnictwa Akademii Teologii Katolickiej, 1995. 
Poźniak Piotr. „La Rue Pierre de.“ Encyklopedia muzyczna PWN. Część biograficzna, Bd. 5: K-L- , hrsg. von Elżbieta Dziębowska, Polskie Wydawnictwo Muzyczne, 1997, S. $293-295$.

Schneider, Peter F. „St. Anna-Messe.“ Edition Biblische Lieder, www.biblische-lieder.de/produkt/stanna-messe-vierstimmiger-chor-und-gemeinde. Abruf am 21.02.2021.

Rozynkowski, Waldemar. „Kult św. Anny w państwie krzyżackim w Prusach.“ Kaplica św. Anny na zamku wysokim w Malborku. Dzieje, wystrój, konserwacja, hrsg. von Janusz Hochleitner, Mariusz Mierzwiński, Muzeum Zamkowe w Malborku, 2016, S. 37-42.

„Santez Anna, o Mamm garet / Peden aveit en dud maru ér brezél.“ Canitiques et musique de sacrée de Bretagne, www.kan-iliz.com/santez-anna-o-mamm-garet-peden-aveit-en-dud-maru-er-brezel. Abruf am 12.02.2021.

Schläpfer, Jürg. Richard Flury (1896-1967). Werkverzeichnis. Zentralbibliothek Solothurn, 1994.

Szweykowski, Zygmunt M. „Wstęp.“ Marcin Mielczewski. Opera Omnia, Bd. 3: Msze koncertujące, hrsg. von Zygmunt M. Szweykowski, Instytut Sztuki Polskiej Akademii Nauk, Musica Iagellonica, 2003, S. 7-19.

Towarek, Piotr. „Maria Magdalena w wielkich formach muzycznych.“ Św. Maria Magdalena w wierze, pobożności, teologii i sztuce - dawniej i dziś. Perspektywa uniwersalna i regionalna, hrsg. von Jacek Jezierski et al., Warmińskie Wydawnictwo Diecezjalne, 2015, S. 143-156.

Towarek, Piotr. „Święta Katarzyna Aleksandryjska w muzycznych opracowaniach mszalnych i oratoryjnych." Święta Katarzyna Aleksandryjska w wierze, pobożności, teologii i sztuce - dawniej $i$ dziś. Perspektywa uniwersalna i regionalna, hrsg. von Jacek Jezierski et al., Warmińskie Wydawnictwo Diecezjalne, 2016, S. 153-165.

Towarek, Piotr, „Wielkie formy muzyczne dedykowane Świętej Annie.“ Święta Anna w wierze, pobożności, teologii i sztuce - dawnej $i$ dziś. Perspektywa uniwersalna i regionalna, hrsg. von Ewelina M. Mączka, Sylwia Mikołajczak, Uniwersytet Warmińsko-Mazurski 2021. In Vorbereitung für den Druck).

„Über mich.“ Edition Biblische Lieder, https://biblische-lieder.de/edition/ueber-mich. Abruf am 21.02. 2021.

Welsh, Jennifer. The Cult of St. Anne in Medieval and Early Modern Europe. Taylor \& Fracis, 2016.

Wierzbowski, Teodor. „Pieśń o św. Annie z końca XV wieku.“ Prace Filologiczne, Nr. 5, 1895, S. $99-105$.

\section{MESSE KOMPOSITIONEN ZU EHREN DER HEILIGEN ANNA. UMRISS DER PROBLEMATIK}

$$
\text { Zusammenfassung }
$$

Die Verehrung der heiligen Anna, die sich im Westen seit dem 14. Jahrhundert entwickelt hat, ist mit liturgischen Werken, einschließlich musikalischer Kompositionen, verbunden. Dazu gehören die Messeproprien: Alleluja-Akklamationen mit Strophen, Sequenzen, und vor allem zahlreiche Motetten (Festa, Senfl, Mouton, de Mantua, Lhéritrier, di Lasso, Charpentier). Der vorliegende Artikel konzentriert sich jedoch nur auf ausgewählte Messen als Musikformen, die mit dem Namen der heiligen Großmutter Jesu verbunden sind. Diese Sammlung umfasst Kompositionen von Künstlern wie Pierre de la Rue, Marcin Mielczewski, Josef Gruber, Kazimierz Garbusiński, Charles Haenni und Richard Flury. Eine interessante Entdeckung waren die Messen aus dem 20. Jahrhundert im Zusammenhang mit dem bretonischen Wallfahrtsort Saint-Anne-d'Auray (Bretagne). In ihnen finden sich Bezüge zum gregoria- 
nischen Gesang und zum bretonischen Kirchenlied (Ropartz, Le Penven, Salaün, Le Marec). Ebenfalls mit dem Namen der Heiligen Anna verbunden sind eine zeitgenössische Messe von Peter F. Schneider, die auf einem deutschen Ordinarium Missae basiert, sowie Kompositionen von polnischen Autoren: Józef Świder und Anna M. Huszcza. Dies sind wichtige Spuren, die die Vitalität des Christentums in der Musikkultur der Vergangenheit und Gegenwart bezeugen.

Schlüsselwörter: Messe; Ordinarium Missae; St. Anna; geistliche Musik.

\section{KOMPOZYCJE MSZALNE KU CZCI ŚWIĘTEJ ANNY. ZARYS PROBLEMATYKI}

Streszczenie

Z rozwijającym się na Zachodzie od XIV w. kultem św. Anny związana została twórczość liturgiczna, w tym kompozycje muzyczne. Chodzi o propria mszalne: aklamacje alleluja z wersetami oraz sekwencje, a przede wszystkim liczne motety (Festa, Senfl, Mouton, de Mantua, Lhéritrier, di Lasso, Charpentier). Przedłożony artykuł skupia się jednak tylko na wybranych mszach jako formach muzycznych związanych z imieniem świętej babki Jezusa. Do zbioru tego należą kompozycje takich twórców jak: Pierre de la Rue, Marcin Mielczewski, Josef Gruber, Kazimierz Garbusiński, Charles Haenni, Richard Flury. Ciekawym odkryciem okazały się dwudziestowieczne oraz współczesne msze związane z bretońskim sanktuarium w Saint-Anne-d'Auray (Bretania). Odnajdujemy w nich nawiązania do chorału gregoriańskiego oraz bretońskiej pieśni religijnej (Ropartz, Le Penven, Salaün, Le Marec). Z imieniem św. Anny złączona została także, oparta o niemieckie ordinarium, współczesna msza Petera F. Schneidera oraz kompozycje polskich twórców: Józefa Świdra i Anny M. Huszczy. Są to ważne ślady świadectwa żywotności chrześcijaństwa w kulturze muzycznej przeszłości i teraźniejszości.

Słowa kluczowe: msza; Ordinarium Missae; św. Anna; muzyka sakralna.

\section{LITURGICAL COMPOSITIONS IN HONOR OF SAINT ANNE. A TOPICAL OVERVIEW}

S u m m a ry

As devotion to St. Anne developed in the West from the 14th century onwards, it became associated with liturgical works, including musical compositions. These include the propria of Mass: alleluia acclamations with verses and sequences, and, above all, numerous motets (Festa, Senfl, Mouton, de Mantua, Lhéritrier, di Lasso, Charpentier). This article, however, focuses only on selected Masses, which, in their musical forms, are associated with the name of Jesus's Holy Grandmother. This collection includes compositions by such artists as Pierre de la Rue, Marcin Mielczewski, Josef Gruber, Kazimierz Garbusiński, Charles Haenni and Richard Flury. An interesting discovery was made regarding 20th-century and contemporary Masses connected with the Breton sanctuary in SaintAnne-d'Auray (Brittany). References to Gregorian chants and Breton religious songs (Ropartz, Le Penven, Salaün, Le Marec) have been found there. Contemporary Masses by Peter F. Schneider, based on a German ordinarium, and compositions by the Polish composers Joseph Świder and Anna M. Huszcza have also been linked to the name of St. Anne. These are all important testimonies to the vitality of Christianity in the musical culture of the past and present.

Keywords: Mass; ordinarium Missae; St. Anne; sacred music. 